\title{
Long-Term Safety and Efficacy of Nonacog Beta Pegol (N9-GP) Administered for at Least 5 Years in Previously Treated Children with Hemophilia B
}

\author{
Manuel Carcao ${ }^{1}$ Susan Kearney ${ }^{2}$ Meng Yao Lu $^{3}$ \\ Elena Santagostino ${ }^{6}$ \\ ${ }^{1}$ Division of Haematology/Oncology, Department of Paediatrics and \\ Child Health Evaluative Sciences, Research Institute, Hospital for Sick \\ Children, University of Toronto, Toronto, Ontario, Canada \\ ${ }^{2}$ Center for Bleeding and Clotting Disorders, Children's Hospital of \\ Minnesota, Minneapolis, Minnesota, United States \\ ${ }^{3}$ Department of Pediatrics, National Taiwan University Hospital, \\ Taipei, Taiwan \\ ${ }^{4}$ Department of Pediatrics, Yokohama City Seibu Hospital, St. \\ Marianna University School of Medicine, Yokohama, Japan \\ ${ }^{5}$ Novo Nordisk A/S, Søborg, Denmark \\ ${ }^{6}$ Foundation IRCCS Cá Granda, Angelo Bianchi Bonomi Hemophilia \\ and Thrombosis Center, Maggiore Hospital Policlinico, Milan, Italy \\ Thromb Haemost 2020;120:737-746.
}

Masashi Taki ${ }^{4}$ Daniel Rubens ${ }^{5}$ Chunduo Shen ${ }^{5}$

\begin{abstract}
Address for correspondence Manuel Carcao, MD, Division of Haematology/Oncology, Department of Paediatrics, Hospital for Sick Children, 555 University Avenue, Room 9416, Toronto, ON M5G 1X8, Canada (e-mail: manuel.carcao@sickkids.ca).
\end{abstract}

\section{Abstract \\ Keywords \\ - N9-GP \\ - hemophilia B \\ - extended half-life \\ - long-term safety \\ - pediatric clinical trial}

Long-term safety and efficacy data of extended half-life factor IX (FIX) prophylaxis in children with hemophilia B (HB) are sparse. paradigm 5 is a multinational, open-label, single-arm, phase III trial assessing once-weekly (40 IU/kg) prophylactic nonacog beta pegol (N9-GP) in previously treated patients (PTPs) aged $\leq 12$ years with HB (FIX activity $\leq 2 \%$ ). Primary endpoint: incidence of anti-FIX inhibitory antibodies $(\geq 0.6$ Bethesda Units). We present a 5-year analysis ( $N=25$, including remaining patients with $\geq 5$ years' follow-up) and compare with a 1-year analysis ( $\geq 52$ weeks' exposure). The main phase enrolled 25 children; 22 entered the extension phase; 17 remained in trial at data cutoff. Median treatment period: 5.6 years/patient; median total number of N9-GP exposure days: 290.0/patient. No patients developed anti-FIX inhibitory antibodies. No other safety concerns, including thromboembolic events, were reported. Neurological examinations have not revealed any new abnormal findings. Sixteen (64.0\%) patients remained free from spontaneous bleeds; all bleeds were mild/moderate in severity; $93.0 \%$ were controlled with 1 to 2 N9-GP injections. No intracranial hemorrhages were reported. Annualized bleeding rates (ABRs) were very low at 5 years (median/Poisson-estimated mean overall ABR: 0.66/0.99), having decreased from the 1-year analysis (1.00/1.44). Median/Poisson-estimated mean spontaneous ABRs for the 1 - and 5-year analyses: $0.00 / 0.45$ and $0.00 / 0.33$. Mean FIX trough activity at 5 years: $17.9 \%$. Mean polyethylene glycol plasma concentration reached steady state at 6 months, increasing slightly over time, in line with increased FIX trough activity. N9-GP administered for $\geq 5$ years shows favorable long-term safety and efficacy in PTPs with HB (FIX activity $\leq 2 \%$ ). received October 11, 2019 accepted February 20, 2020
DOI https://doi.org/ $10.1055 / \mathrm{s}-0040-1709521$. ISSN 0340-6245. (c) 2020 Georg Thieme Verlag KG Stuttgart . New York
License terms

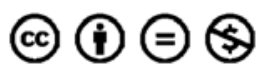




\section{Introduction}

Severe hemophilia B (factor IX [FIX] activity $<1 \%$ ) is associated with frequent spontaneous bleeds, particularly joint and musculoskeletal bleeding, leading to chronic pain and disability. Additionally, patients with severe hemophilia B are at risk of life-threatening bleeding, such as intracranial hemorrhage (ICH). ${ }^{1-3}$ Primary prophylaxis, defined as initiation of regular factor concentrate infusions in young children (generally between 1 and 2 years of age) prior to a joint bleed, has become the recommended approach for the management of severe hemophilia $\mathrm{B}^{4-6}$

In the past, the goal of prophylactic therapy in severe hemophilia B has been to maintain plasma FIX activity above $1 \%$, based on evidence that patients with moderate hemophilia B (FIX activity of 1-5\%) experience significantly fewer bleeds and their associated complications. ${ }^{4}$ Historically, to achieve this threshold, standard FIX (recombinant or plasma-derived) products have been administered at least twice per week. However, the need for such frequent infusions can negatively affect quality of life, adherence to treatment, and, ultimately, long-term treatment efficacy. ${ }^{1,4,7}$ Frequent administration is problematic for young children in whom venous access is particularly challenging and who often require insertion of central venous access devices (CVADs). Such devices, in addition to requiring surgery and hospitalization to insert, are associated with several complications, including malfunction, infection, and thrombosis. ${ }^{8}$

To address the challenges associated with frequent dosing, an extended half-life beta EHL) FIX, nonacog beta pegol (N9-GP; REFIXIA/REBINYN, Novo Nordisk A/S, Bagsværd, Denmark), has been developed. N9-GP employs site-specific glyco-PEGylation on the activation peptide of FIX, which has been shown to prolong FIX half-life by up to fivefold over standard half-life recombinant or plasma-derived FIX. ${ }^{9}$ Clinical efficacy of N9-GP has been confirmed in three trials involving previously treated adults and adolescents with severe or moderately severe hemophilia B (FIX activity $\leq 2 \%$ ). ${ }^{10-12}$

The paradigm 5 trial was initiated in 2012 to assess the safety and efficacy of once-weekly N9-GP prophylaxis (40 $\mathrm{IU} / \mathrm{kg}$ ) in previously treated children (aged $\leq 12$ years) with hemophilia B and FIX activity $\leq 2 \%$. The first interim analysis, performed in 2014 when the last enrolled patient had reached 50 exposure days (EDs), demonstrated safety and efficacy in this population over a $\geq 1$-year period of follow-up data. ${ }^{13}$ However, long-term safety and efficacy data are of particular importance in young children, given the anticipated duration of exposure. Here, we present long-term safety and efficacy data from the paradigm 5 trial after a minimum of 5 years of follow-up and compare results with the 1-year analysis. ${ }^{13}$

\section{Methods}

\section{Trial Design}

The design of paradigm 5 has previously been described in detail. ${ }^{13}$ Briefly, paradigm 5 is a multinational, open-label, single-arm, phase III trial (ClinicalTrials.gov identifier: NCT01467427) with a 52-week main phase (initiated in
May 2012) and an ongoing extension phase. The trial seeks to follow patients over a 10-year follow-up period (-Supplementary Fig. S1, available in the online version). The data cutoff date for this current 5-year analysis was October 1, 2018.

All patients received once-weekly intravenous N9-GP 40 $\mathrm{IU} / \mathrm{kg}$ prophylaxis during the main and extension phases. Uncomplicated mild/moderate bleeding episodes, such as joint bleeds, were to be treated with a single dose of N9-GP $40 \mathrm{IU} / \mathrm{kg}$ and severe bleeding episodes (such as ICHs) were to be treated with a single dose of $80 \mathrm{IU} / \mathrm{kg}$. For placement of CVADs (ports) or minor surgeries, an additional dose of N9-GP $40 \mathrm{IU} / \mathrm{kg}$ was administered. Major surgery was permitted following a protocol amendment in July 2014 and conducted in accordance with the World Federation of Hemophilia guidelines. ${ }^{4}$

If high-titer ( $\geq 5$ Bethesda Units [BU]) anti-FIX inhibitory antibodies were confirmed or if significant clinical impact from anti-FIX inhibitory antibodies was observed, the patient was to be withdrawn from N9-GP treatment. In the event of low-titer ( $<5 \mathrm{BU}$ ) anti-FIX inhibitory antibodies, the patient could remain in the trial.

\section{Patients}

Key inclusion criteria for paradigm 5 included male gender, age $\leq 12$ years, moderately severe or severe congenital hemophilia B (FIX activity $\leq 2 \%$ ), and a history of $\geq 50$ EDs to other FIX products. Informed consent from the patients' parents/caregivers was obtained before any trial-related activities. Key exclusion criteria included a known history of anti-FIX inhibitory antibodies and an anti-FIX inhibitory antibody level of $\geq 0.6$ BU at screening.

\section{Endpoints}

Safety: The primary endpoint was incidence of anti-FIX inhibitory (neutralizing) antibodies, defined as $\geq 0.6$ BU. Secondary safety endpoints included the incidence of adverse events (AEs), serious AEs (SAEs), medical events of special interest (MESIs), and development of anti-Chinese hamster ovary $(\mathrm{CHO})$ antibodies. The following were considered to be MESIs: medication errors of trial product, development of anti-FIX inhibitory antibodies, thromboembolic events, anaphylactic reactions, allergic reactions, and central nervous system (CNS)-related AEs. General safety was also assessed. A neurological examination was added to the investigational plan in December 2017. This included general appearance; language, social, and developmental aspects; handedness; head circumference; level of consciousness; cranial nerves in relation to sight (reaction to light, visual fields and acuity, and eye movements); hearing; muscle function (neck, upper and lower extremities); sensory aspect of cold, pin prick, light touch and proprioception; and gait (walking, running, on heels and toes, standing or hopping on one leg, and Romberg's test).

In addition, exploratory plasma polyethylene glycol (PEG) concentration was measured. Blood samples collected since the start of the paradigm 5 trial in 2012 were identified and, following receipt of informed consent, analyzed for PEG plasma levels. After the protocol amendment (December 2017), 
blood samples were prospectively collected and analyzed for plasma PEG levels.

Efficacy: Efficacy endpoints were all secondary and included the number of bleeding episodes during prophylaxis (annualized bleeding rate [ABR]), type of bleed, bleed location, number of N9-GP injections needed to treat a bleed, and hemostatic control of bleeding, assessed on a four-point hemostatic response scale. An "excellent" response was defined as abrupt pain relief and/or clear improvement in objective signs of bleeding within 8 hours after a single infusion; a "good" response as noticeable pain relief and/or improvement in signs of bleeding within 8 hours after a single injection; a "moderate" response as probable or slight beneficial effect within the first 8 hours after the first injection but requiring more than one injection within 8 hours; and a "poor" response as no improvement or worsening of symptoms within 8 hours after two injections. Successful control of bleeding was defined as either an "excellent" or a "good" response. In addition, the amount of N9-GP administered for purposes of prophylaxis, bleed management, and minor or major surgery was reported.

Pharmacokinetic (PK) parameters: Steady-state FIX trough activity (\%) was measured at each clinic visit. The PK analysis was performed with a one-stage clot assay, based on a modified activated partial thromboplastin time assay and using a product-specific calibrator.

Health economic resource use: Endpoints included duration of hospitalization, number of preschool/school days missed due to bleeds or their sequelae, and number of workdays missed by parent(s)/caregiver(s) due to bleeds or their sequelae.

\section{Antibody Testing}

Detection of anti-FIX inhibitory antibodies (titer of $\geq 0.6 \mathrm{BU}$ ) was performed using a modified Nijmegen FIX Bethesda assay. Samples were analyzed at a central laboratory, and, if positive, a second confirmatory assay was performed. ${ }^{13}$ In the main phase, blood samples to be tested for anti-FIX inhibitory antibodies were collected predose and 7 days after the first dose, then every 4 weeks up to week 12 , and then every 8 weeks up to and including week 52 . In the extension phase, testing for anti-FIX inhibitory antibodies was performed at 3 months after the end of the main phase and then every 6 months thereafter. Screening for N9-GP-binding antibodies and FIX-binding antibodies in plasma was conducted using a bridging enzyme-linked immunosorbent assay (ELISA). ${ }^{14}$ Anti-PEG antibodies were not directly measured; any antibodies binding to N9-GP that did not crossreact to recombinant FIX ( $r F I X)$ were assumed to be directed against the PEG domain. Anti-CHO antibodies were measured using a direct ELISA approach. ${ }^{15}$

\section{Statistical Analysis}

The sample size was based on European Medicines Agency guidelines. ${ }^{16}$ At enrollment, patients were stratified into two age groups (0-6 and 7-12 years), with the aim of $\geq 10$ patients in each age group completing the main phase with $\geq 50$ EDs for statistical evaluation.
Confidence intervals (Cls) for the anti-FIX inhibitory antibody rate and the ABR were determined; assessments of all other endpoints were based on descriptive analyses. All summaries and analyses were performed for the total group, as well as for each age group. The full analysis and safety analysis sets were identical and comprised all patients exposed to N9-GP. All data reported were cumulative.

For the primary endpoint, the incidence of anti-FIX inhibitory antibodies was reported, and a one-sided $97.5 \%$ upper confidence limit was determined, based on an exact calculation for a binomial distribution. Adequate safety, with respect to anti-FIX inhibitory antibodies, was concluded if the observed rate was $\leq 5 \%$, corresponding to no more than one patient with anti-FIX inhibitory antibodies.

ABRs (overall, spontaneous, traumatic, or joint) were reported and a two-sided $95 \% \mathrm{CI}$ was provided based on a Poisson regression model allowing for overdispersion. A Poisson regression model was chosen because it adjusts for the different exposure times of the patients in trial, whereas the conventional mean ABR treats all exposure times equally. Consequently, the Poisson regression model provides a more accurate estimate of ABR.

The analysis of mean FIX trough activity at steady state during prophylaxis was based on a mixed model on the logtransformed plasma concentrations with patient as a random effect. Only predose FIX measurements taken at least 5 days and no more than 10 days after the last dose, as well as measurements taken at least 14 days after the last bleeding episode, were included in the calculation of steady-state FIX trough activity.

\section{Results}

As previously described, 25 patients were enrolled in this trial from 17 sites in 8 countries. ${ }^{13}$ Of these 25 patients, 24 completed the main phase and 22 entered the extension phase. Some attrition was expected given the length of the trial and the increasing age of the patients. At data cutoff for the 5-year analysis, 17 patients remained in the trial, while 8 had discontinued; one patient did not complete the main phase, two patients did not enter the extension phase of the trial, and five discontinued during the extension phase. For the five patients who withdrew during the extension phase, reasons for withdrawal included unwillingness to follow trial procedures (one patient), parental anxiety over PEGylated product (one patient), relocation of principal investigators (one patient), and deciding to change treatment regimen (two patients). No patients were withdrawn due to AEs. At baseline, median (range) age was $7.0(1-12)$ years in the overall population. - Table 1 shows additional patient characteristics at baseline. At the time of this 5 -year analysis, median (range) age was 11.1 (6-18) years in the 17 remaining patients: $9.2(6-12)$ years in the younger age group and 15.7 (14-18) years in the older age group.

\section{N9-GP Exposure}

The 5-year analysis (including the main and extension phases) comprised 116.44 patient years and 6,194 N9-GP EDs. The median (range) total number of EDs per patient was 
Table 1 Patient demographics at baseline ${ }^{13}$

\begin{tabular}{|l|l|l|l|}
\hline & Younger children (0-6 y) & Older children (7-12 y) & Total \\
\hline Number of patients & 12 & 13 & 25 \\
\hline Age (y) & \multicolumn{2}{|l|}{} \\
\hline Mean (SD) & $3.1(1.7)$ & $9.6(1.6)$ & $6.5(3.7)$ \\
\hline Median (min-max) & $3.0(1-6)$ & $10.0(7-12)$ & $7.0(1-12)$ \\
\hline \multicolumn{5}{|l|}{ Race, $N$ (\%) } & $4(33.3)$ & $4(30.8)$ & $8(32.0)$ \\
\hline Asian & - & $1(7.7)$ & $1(4.0)$ \\
\hline Black/African American & $8(66.7)$ & $5(38.5)$ & $13(52.0)$ \\
\hline White & - & $3(23.1)$ & $3(12.0)$ \\
\hline Other & \multicolumn{3}{l}{} \\
\hline BMI (kg/m $\left.{ }^{2}\right)$ & $17.0(1.7)$ & $18.0(2.1)$ & $17.5(2.0)$ \\
\hline Mean (SD) & $17.0(14.7-21.1)$ & $17.5(15.3-23.3)$ & $17.5(14.7-23.3)$ \\
\hline Median (min-max)
\end{tabular}

Abbreviations: BMI, body mass index; $N$, number of patients; SD, standard deviation.

${ }^{a}$ No patients were $<1$ year of age at baseline.

290.0 (10.0-325.0), while the median (range) number of prophylactic N9-GP injections was 279.0 (10.0-318.0).

\section{Safety}

Immunogenicity: No patients developed anti-FIX inhibitory (neutralizing) antibodies (primary endpoint) over the 5-year period analyzed. As previously reported, one patient was transiently positive (visit 11) for anti-CHO antibodies in the main phase of the trial without clinical consequences; the patient remained in the trial. ${ }^{13}$ In the extension phase, one patient with a small deletion FIX mutation (currently aged 16 years) was transiently positive for a binding, non-neutralizing antibody to N9-GP, which cross-reacted against standard rFIX. The antibody did not have any clinical impact (no impact on PK parameters or AEs and no increase in bleeding rate). Currently (post data cutoff), the patient is negative for N9-GP-binding antibodies. No anti-PEG antibodies were detected in any patients.

Adverse events: Over the entire 5-year trial period, 24 (96.0\%) patients reported 573 AEs (4.92 AEs per patient-year of exposure) (-Table 2). Of these, four (occurring in three patients) were considered to be SAEs (catheter-site infection, viral upper respiratory tract infection, food poisoning, and hemoptysis). An additional three SAEs were reported but were linked to existing SAEs. Importantly, no thromboembolic events or severe allergic reactions were reported.

Adverse events considered possibly/probably related to trial product: A total of eight AEs occurring in four patients (three patients in the 7-12-year age group accounted for six of these AEs; one patient in the $0-6$-year age group accounted for two of these AEs) were determined by site investigators to be possibly/probably related to trial product, corresponding to 0.07 events per patient-year of exposure (-Table 2 ). These were abdominal pain, diarrhea, administration-site pain (two AEs), eosinophilia, headache, nausea, and wheezing; all occurred during the first year of the trial and did not result in product discontinuation. No SAEs were judged by the investigators to be related to N9-GP.
Medical events of special interest: A total of seven MESIs (0.06 MESIs per patient-year of exposure) in five patients were reported, of which six were allergic reactions (-Table 2). These were urticaria (two episodes in one patient), miliaria, allergic rhinitis, wheezing, and allergy to arthropod bite. Only wheezing was considered possibly/probably related to the trial drug. One patient experienced a CNS-related event (Tourette's disorder) that was not judged to be related to the trial drug, and he remained on treatment.

Neurological assessments: Fifteen patients underwent the first structured neurological examination. No patients displayed any new abnormal findings. Three patients had abnormal neurological findings that had already been reported prior to the first structured neurological examination, and none were assessed as being related to the trial or to N9-GP. One of these patients had abnormal social interaction and language skills and was not reaching developmental milestones. This patient also had a previous ICH at birth and, while in the trial, had been diagnosed with attention-deficit hyperactivity disorder; this was considered unlikely to be related to treatment. Another patient had preexisting reduced visual acuity, and a third patient had gait issues at baseline due to a target joint in his right foot. By the data cutoff date for this analysis, two patients had undergone a second annual neurological examination and no abnormalities were reported.

Laboratory findings: Mean PEG plasma concentration reached steady state at 6 months, after which a slight increase occurred over $\geq 5$ years, in line with the increase in FIX trough activity; this corresponded to the expected decrease in FIX clearance, which occurs with increasing patient age ( - Fig. 1A, B). As expected, with increasing age, creatinine levels also increased slightly over the trial period but remained age appropriate and within the reference range. Liver enzymes remained stable over time. No safety concerns were identified. 
Table 2 Safety outcomes at 1- and 5-year analyses

\begin{tabular}{|c|c|c|c|c|}
\hline & \multirow{2}{*}{$\begin{array}{l}\text { 1-year analysis }{ }^{13} \\
(n=25)\end{array}$} & \multicolumn{3}{|c|}{ 5-year analysis $(n=25)^{a}$} \\
\hline & & $\begin{array}{l}\text { Younger children } \\
(0-6 \text { y) } \\
(n=12)\end{array}$ & $\begin{array}{l}\text { Older children } \\
(7-12 y) \\
(n=13)\end{array}$ & $\begin{array}{l}\text { Total } \\
(n=25)\end{array}$ \\
\hline \multicolumn{5}{|l|}{ N9-GP exposure } \\
\hline Sum of years in trial for all patients & 29.17 & 57.04 & 59.40 & 116.44 \\
\hline EDs in trial & 1,584 & 3,016 & 3,178 & 6,194 \\
\hline \multicolumn{5}{|l|}{ Immunogenicity, number of patients } \\
\hline Anti-FIX inhibitory Abs & 0 & 0 & 0 & 0 \\
\hline Anti-CHO Abs & 1 & 0 & 0 & 1 \\
\hline N9-GP-binding Abs & 0 & 0 & $1^{\mathrm{b}}$ & $1^{\mathrm{b}}$ \\
\hline Anti-PEG Abs & 0 & 0 & 0 & 0 \\
\hline \multicolumn{5}{|l|}{ Number of AEs/number of patients } \\
\hline All AEs & $250 / 23$ & $303 / 12$ & $270 / 12$ & $573 / 24$ \\
\hline Serious AEs & $1 / 1$ & $2 / 1$ & $2 / 2$ & $4 / 3$ \\
\hline Severe AEs & 0 & $2 / 1$ & $1 / 1$ & $3 / 2$ \\
\hline Moderate AEs & $10 / 7$ & $34 / 6$ & $13 / 7$ & $47 / 13$ \\
\hline Mild AEs & $240 / 23$ & $267 / 12$ & $256 / 12$ & $523 / 24$ \\
\hline \multicolumn{5}{|c|}{ Most commonly reported AEs (number of AEs/number of patients) } \\
\hline Nasopharyngitis & $11 / 5$ & $27 / 6$ & $5 / 2$ & $32 / 8$ \\
\hline Cough & $17 / 10$ & $25 / 10$ & $4 / 4$ & $29 / 14$ \\
\hline Contusion & $17 / 8$ & $12 / 4$ & $16 / 6$ & $28 / 10$ \\
\hline Pyrexia & $14 / 6$ & $19 / 7$ & $7 / 4$ & $26 / 11$ \\
\hline $\begin{array}{l}\text { AEs judged as possibly/probably } \\
\text { related to treatment by investigator }\end{array}$ & $8 / 4$ & $2 / 1$ & $6 / 3$ & $8 / 4$ \\
\hline MESIs & $2 / 2$ & $1 / 1$ & $6 / 4$ & $7 / 5$ \\
\hline
\end{tabular}

Abbreviations: Ab, antibody; AE, adverse event; $\mathrm{CHO}$, Chinese hamster ovary; ED, exposure day; FIX, factor IX; MESI, medical event of special interest; $n$, number of patients; N9-GP, nonacog beta pegol; PEG, polyethylene glycol.

${ }^{\mathrm{a} A E s}$ at the 1- and 5-year analyses (cumulative).

${ }^{\mathrm{b}}$ Cross-reactive against recombinant FIX, transient.

\section{Efficacy}

Bleeding during prophylaxis: In the 1-year analysis, 15 of 25 patients enrolled in the main phase had experienced 42 bleeds requiring treatment ${ }^{13}$; at the 5 -year analysis, an additional five patients had experienced treatment-requiring bleeds over the subsequent 4 years ( - Table 3 ). Therefore, over $\geq 5$ years, 20 patients (80.0\%) had experienced 115 bleeds requiring treatment, with the cause of bleeds being traumatic $(73 ; 63.5 \%$ of bleeds), spontaneous $(38 ; 33.0 \%)$, and other $(4 ; 3.5 \%)$.

Sites of bleeds with reported information on location ( $n=113$ ) were joint $(48 ; 42.5 \%$ of bleeds), muscular (22; $19.5 \%)$, subcutaneous $(19 ; 16.8 \%)$, mucosal $(15 ; 13.3 \%)$, gastrointestinal $(1 ; 0.9 \%)$, or other $(8 ; 7.1 \%)$; location was not reported for two bleeds. No ICHs were reported. All bleeds that occurred were classified as mild or moderate, $88.7 \%$ were successfully controlled with N9-GP ("excellent" or "good" response on the four-point hemostatic response scale), and the majority (93.0\%) were successfully treated with one or two N9-GP injections (-Table 3).
Prophylactic efficacy: Over a median (range) treatment period per patient of $5.6(0.2-6.1)$ years, 5 (20\%) patients remained completely bleed free, $10(40.0 \%)$ patients did not experience any joint bleeds, and 16 (64.0\%) patients experienced no spontaneous bleeds.

The median overall ABR had decreased at the 5-year analysis (0.66) compared with the 1-year analysis (1.00), as had the median traumatic ABR (0.47 vs. 0.68). Median spontaneous ABR was 0.00 at both the 1 - and 5-year analyses, and median joint ABR was 0.00 at the 1 -year analysis and 0.18 at the 5 -year analysis.

The Poisson-estimated mean overall ABR was low in the 1-year analysis (1.44) and continued to decline over the longer observation period in the 5-year analysis period (0.99). Similar findings were observed with the Poisson-estimated mean spontaneous ABR (0.41 decreasing to 0.33 ), mean traumatic ABR (0.89 decreasing to 0.63 ), and mean joint $A B R$ (0.55 decreasing to 0.42). ${ }^{13}$ See - Fig. 2 for median ABR and - Table 4 for median and Poisson-estimated mean ABR outcomes. 

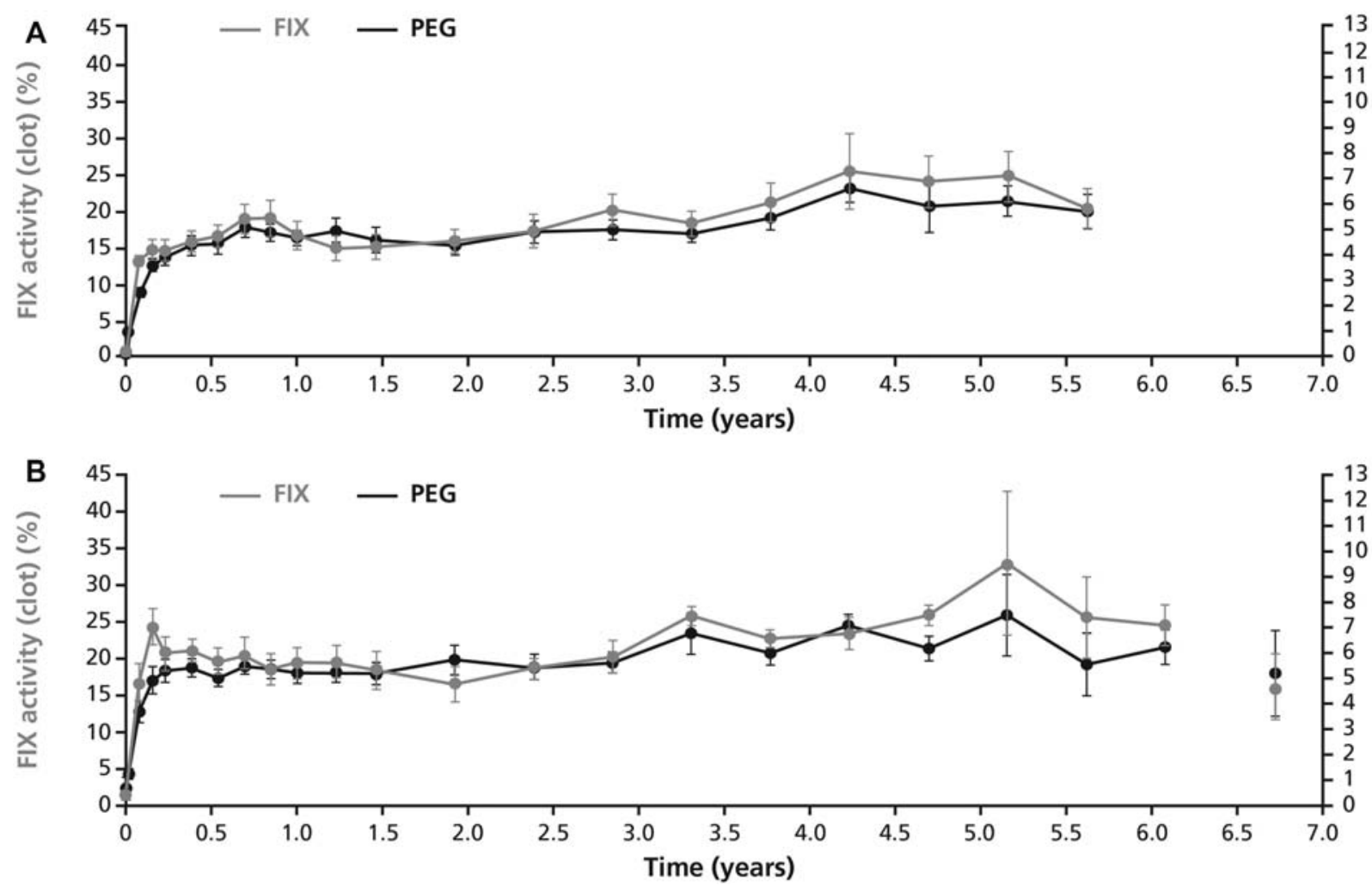

Fig. 1 Mean (SEM) profiles of PEG concentration $(\mu \mathrm{g} / \mathrm{mL})$ and FIX trough activity $(\%)$ for (A) patients aged 0-6 years at start of trial and (B) 7-12 years at start of trial over a follow-up period of at least 5 years. ${ }^{\text {a }}$ FIX, factor IX; PEG, polyethylene glycol; SEM, standard error of the mean. ${ }^{\text {a Points }}$ are means; error bars represent SEM. Profiles are overlaid to reveal patterns between both measurements. Note that different scales are used for each curve. Only predose FIX measurements taken at least 5 days and no more than 10 days after the last dose, as well as measurements taken at least 14 days after the last bleeding episode, were included. The final assessment in the 7- to 12-year age group denotes the end-of-trial visit. This visit was for patients who did not proceed into the extension phase or who were withdrawn.

Table 3 Hemostatic effectiveness of N9-GP at 1- and 5-year analyses ${ }^{\mathrm{a}}$

\begin{tabular}{|c|c|c|c|c|}
\hline & \multirow[t]{2}{*}{$\begin{array}{l}\text { 1-year analysis }{ }^{13} \\
(n=25)\end{array}$} & \multicolumn{3}{|l|}{$\begin{array}{l}\text { 5-year analysis }{ }^{b} \\
(n=25)\end{array}$} \\
\hline & & $\begin{array}{l}\text { Younger children } \\
(0-6 \text { y) } \\
(n=12)\end{array}$ & $\begin{array}{l}\text { Older children } \\
(7-12 \text { y) } \\
(n=13)\end{array}$ & $\begin{array}{l}\text { Total } \\
(n=25)\end{array}$ \\
\hline \multicolumn{5}{|c|}{ Hemostatic response, $N$ (\% treated bleeds) } \\
\hline Excellent & $22(52.4)$ & $21(53.8)$ & $22(28.9)$ & $43(37.4)$ \\
\hline Good & $17(40.5)$ & $14(35.9)$ & $45(59.2)$ & $59(51.3)$ \\
\hline Moderate & $2(4.8)$ & $2(5.1)$ & $7(9.2)$ & $9(7.8)$ \\
\hline Poor & $1(2.4)$ & $2(5.1)$ & $2(2.6)$ & $4(3.5)$ \\
\hline \multicolumn{5}{|c|}{ Number of injections per bleeding episode, $N(\%)$} \\
\hline 1 & $36(85.7)$ & $33(84.6)$ & $61(80.3)$ & $94(81.7)$ \\
\hline 2 & $5(11.9)$ & $5(12.8)$ & $8(10.5)$ & $13(11.3)$ \\
\hline$\geq 3$ & $1(2.4)$ & $1(2.6)$ & $7(9.2)$ & $8(7.0)$ \\
\hline \multicolumn{5}{|c|}{ Successful control of bleeding ${ }^{\mathrm{c}}, N$ (\% treated bleeds) } \\
\hline Overall & $39(92.9)$ & $35(89.7)$ & $67(88.2)$ & $102(88.7)$ \\
\hline Traumatic & $24(96.0)$ & $28(90.3)$ & $38(90.5)$ & $66(90.4)$ \\
\hline Spontaneous & $11(84.6)$ & $7(87.5)$ & $25(83.3)$ & $32(84.2)$ \\
\hline
\end{tabular}

Abbreviations: $n$, number of patients; $N$, total number of bleeding episodes; N9-GP, nonacog beta pegol.

${ }^{\mathrm{a} E v a l u a t i o n s ~ i n c l u d e ~ o n l y ~ t h o s e ~ b l e e d s ~ r e q u i r i n g ~ t r e a t m e n t . ~}$

bIncludes data from the 1-year analysis.

cDetermined as being either an excellent or a good response. 


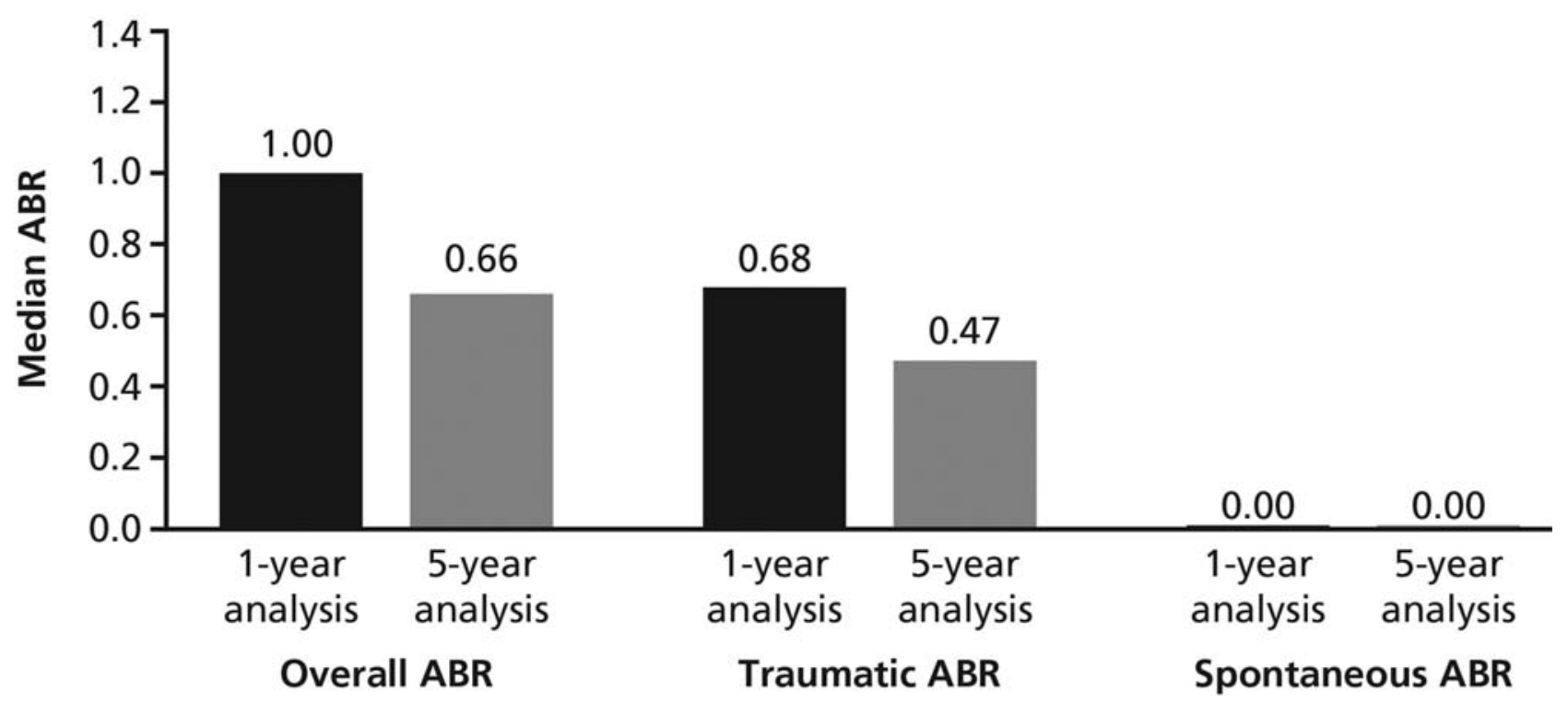

Fig. 2 Changes in median overall, traumatic, and spontaneous ABRs at 1-year analysis versus 5-year analysis (total population). ${ }^{13}$ ABR, annualized bleeding rate.

N9-GP consumption: Median (range) annual total N9-GP consumption was 2288.1 (2015.1-2512.4) IU/kg/year per patient, median (range) annual prophylactic N9-GP consumption was 2220.6 (2015.1-2333.6) IU/kg/year per patient, and median (range) annual N9-GP consumption for treatment of bleeds was 50.6 (7.7-374.9) IU/kg/year per patient. Median (range) N9-GP dose per bleeding episode was 43.2 (21.9-569.3) IU/kg/bleed and the median (range) prophylactic dose was 43.1 (6.4-56.0) IU/kg.

One patient underwent major surgery for closure of arteriovenous fistula; he received two N9-GP doses of 41.8 $\mathrm{IU} / \mathrm{kg}$ on the day of surgery. Ten patients underwent 17 minor surgeries (dental procedures $[n=10]$, CVAD removal/insertion [ $n=3$ ], bronchoscopy [ $n=1]$, nasal deviation correction $[n=1]$, myringotomy and grommet (ear tube) insertion $[n=1]$, and grommet insertion $[n=1]$ ). Patients undergoing minor surgery received a median (range) N9-GP dose of 44.1 (40.6-61.5) IU/kg/procedure.

\section{Health Economic Outcomes}

At data cutoff, most children had missed no days of school due to bleeds or their sequelae ( 81.8 and $84.6 \%$ of children aged 0-6 and 7-12 years, respectively) and none of the children spent any time as inpatients in hospital, outside of surgery settings. Over the 5-year analysis period, parents of children in the 0 to 6 - and 7 to 12-year age groups missed a mean of 0.3 and 0.5 days of work, respectively, due to a child's bleed or its sequelae.

\section{Pharmacokinetics}

Mean FIX trough activity increased slightly over the analysis period ( - Fig. 1A, B). By the fifth year on trial, the estimated mean percentage steady-state FIX trough activity (95\% CI) was $17.9 \%$ (15.8-20.3\%) in the overall patient group (median age at analysis: 11.1 years), $16.6 \%(13.8-19.9 \%)$ in the younger age group (median age at analysis: 9.2 years), and $19.2 \%$
(16.1-22.9\%) in the older age group (median age at analysis: 15.7 years).

\section{Discussion}

In this 5-year analysis, long-term safety and efficacy of prophylactic N9-GP administered as $40 \mathrm{IU} / \mathrm{kg}$ once weekly were established. All 17 patients still in trial at the time of this analysis had at least 5 years of follow-up data.

No safety concerns were identified, and no patients developed anti-FIX inhibitory antibodies during this longterm follow-up period. Although there is extensive clinical experience with PEGylated molecules, there has been some debate regarding whether abnormal accumulation of PEG in plasma and tissues may occur during long-term treatment and whether this might impact the functioning of processing organs, such as the kidneys and liver. Analysis of patient samples from this study over a treatment duration of up to 6.5 years demonstrated that PEG plasma concentration reached steady state at 6 months, after which there was only a slight increase over the analysis period ( $\geq 5$ years), correlating closely with a gradual increase in FIX trough activity over the same period. ${ }^{17}$ This increase was expected given that FIX clearance decreases as children grow older and assume adult-like metabolism ${ }^{3}$; in fact, at the time of this analysis, 10 patients were already adolescents ( $\geq 12$ years). These data, combined with data from adolescents and adults with hemophilia B receiving weekly prophylactic N9-GP $(40 \mathrm{IU} / \mathrm{kg})$, were recently published in an independent manuscript that focused on steady-state PEG plasma levels during long-term prophylactic treatment. It demonstrated that PEG plasma levels followed predictable kinetics and reached steady state in children, adolescents, and adults on regular prophylaxis with N9-GP, with no long-term accumulation observed in up to 6.5 years of regular prophylaxis. ${ }^{17}$ Moreover, Sternebring et al discuss experiences from other 
Table 4 Treatment outcomes at 1- and 5-year analyses

\begin{tabular}{|c|c|c|c|c|}
\hline & \multirow[b]{2}{*}{$\begin{array}{l}\text { 1-year analysis }{ }^{13} \\
(n=25)\end{array}$} & \multicolumn{3}{|l|}{$\begin{array}{l}\text { 5-year analysis }{ }^{a} \\
(n=25)\end{array}$} \\
\hline & & $\begin{array}{l}\text { Younger children } \\
(0-6 \text { y) } \\
(n=12)\end{array}$ & $\begin{array}{l}\text { Older children } \\
(7-12 y) \\
(n=13)\end{array}$ & $\begin{array}{l}\text { Total } \\
(n=25)\end{array}$ \\
\hline \multicolumn{5}{|l|}{ Treatment period per patient, y } \\
\hline Mean (SD) & $1.2(0.3)$ & $4.8(1.7)$ & $4.6(1.9)$ & $4.7(1.8)$ \\
\hline Median (min-max) & $1.2(0.2-1.5)$ & $5.6(0.2-5.7)$ & $5.3(1.0-6.1)$ & $5.6(0.2-6.1)$ \\
\hline \multicolumn{5}{|l|}{ Efficacy outcomes ${ }^{b}$} \\
\hline Number of bleeds/patients with bleeds & $42 / 15$ & $39 / 8$ & $76 / 12$ & $115 / 20$ \\
\hline Number of patients without a bleed (\%) & $10(40.0)$ & $4(33.3)$ & $1(7.7)$ & $5(20.0)$ \\
\hline $\begin{array}{l}\text { Number of patients without } \\
\text { a spontaneous bleed (\%) }\end{array}$ & $19(76.0)$ & $9(75.0)$ & $7(53.8)$ & $16(64.0)$ \\
\hline \multicolumn{5}{|l|}{ Bleed cause, $N(\%)$} \\
\hline Traumatic & $25(59.5)$ & $31(79.5)$ & $42(55.3)$ & $73(63.5)$ \\
\hline Spontaneous & $13(31.0)$ & $8(20.5)$ & $30(39.5)$ & $38(33.0)$ \\
\hline Other/unknown & $4(9.5)$ & 0 & $4(5.3)$ & $4(3.5)$ \\
\hline \multicolumn{5}{|l|}{ Median ABR (IQR) } \\
\hline Overall ABR & $1.00(0.00-2.06)$ & $0.41(0.00-1.13)$ & $0.99(0.33-2.68)$ & $0.66(0.33-1.80)$ \\
\hline $\mathrm{ABR}_{\text {traumatic }}$ & $0.68(0.00-1.03)$ & $0.41(0.00-0.76)$ & $0.50(0.33-0.82)$ & $0.47(0.17-0.82)$ \\
\hline $\mathrm{ABR}_{\text {spontaneous }}$ & $0.00(0.00-0.00)$ & $0.00(0.00-0.18)$ & $0.00(0.00-0.75)$ & $0.00(0.00-0.36)$ \\
\hline $\mathrm{ABR}_{\text {joint }}$ & $0.00(0.00-0.80)$ & $0.18(0.00-0.37)$ & $0.17(0.00-0.82)$ & $0.18(0.00-0.54)$ \\
\hline \multicolumn{5}{|l|}{ Poisson-estimated mean ABR $(95 \% \mathrm{Cl})^{c}$} \\
\hline Overall ABR & $1.44(0.92-2.26)$ & $0.68(0.32-1.48)$ & $1.28(0.74-2.23)$ & $0.99(0.61-1.60)$ \\
\hline $\mathrm{ABR}_{\text {traumatic }}$ & $0.86(0.50-1.47)$ & $0.54(0.23-1.27)$ & $0.71(0.34-1.47)$ & $0.63(0.36-1.10)$ \\
\hline $\mathrm{ABR}_{\text {spontaneous }}$ & $0.45(0.18-1.08)$ & $0.14(0.03-0.60)$ & $0.51(0.24-1.07)$ & $0.33(0.15-0.70)$ \\
\hline $\mathrm{ABR}_{\text {joint }}$ & $0.55(0.30-1.00)$ & $0.25(0.11-0.53)$ & $0.59(0.36-0.96)$ & $0.42(0.26-0.68)$ \\
\hline
\end{tabular}

Abbreviations: $A B R$, annualized bleeding rate (bleeds per patient per year); $A_{B R} R_{\text {joint }}$, annualized bleeding rate in any joint; $A B R_{\text {spontaneous, }}$ annualized spontaneous bleeding rate; $\mathrm{ABR}_{\text {traumatic }}$, annualized traumatic bleeding rate; $\mathrm{Cl}$, confidence interval; IQR, interquartile range; $n$, number of patients; $\mathrm{N}$, total number of bleeding episodes; SD, standard deviation.

ancludes data from the 1-year analysis.

bEvaluations include only those bleeds requiring treatment.

${ }^{\mathrm{c}}$ Analyzed based on a Poisson-regression analysis model allowing for overdispersion and using treatment duration as an effect.

PEGylated products in long-term use that reach similar or higher steady-state PEG plasma levels than those achieved with N9-GP prophylaxis. There has been no indication that these steady-state exposures to PEG caused any safety concerns or unexpected AEs. ${ }^{17}$ These findings are in line with those from an earlier study by Stidl et al, which also concluded that there is no consistent pattern of AEs attributable to PEG exposure for any of several PEGylated drugs evaluated (including N9-GP and FVIII/FIX replacement therapies that include PEG as an excipient). ${ }^{18}$

In the current study, no new abnormal neurological findings were reported in the 15 patients who had undergone a neurological examination, and there were no clinically significant changes in the levels of creatinine or liver enzymes over the 5-year analysis period, demonstrating a lack of neurologic, renal, and hepatic toxicity. As such, despite 5 years of ongoing regular PEG exposure, no safety concerns have been identified so far. Moreover, the overall experience from N9-GP clinical trials thus far demonstrates that the safety profile of N9-GP is similar to other nonPEGylated FIX products and no safety issues attributable to PEG have been identified. ${ }^{9-13}$

In addition to showing robust long-term safety, N9-GP was associated with favorable efficacy outcomes over a follow-up period of at least 5 years. It is noteworthy that ABRs diminished from the initial 1-year analysis to the 5year analysis; this included median overall and traumatic ABRs ( 1.00 vs. 0.66 and 0.68 vs. 0.47 , respectively) as well as Poisson-estimated mean ABR measures (overall, spontaneous, traumatic, and joint). Median spontaneous ABRs did not change as they were 0.00 for both analyses. There are several possible reasons to explain the fall in bleeding rates over time. In the first year on trial, patients/families lacking familiarity with N9-GP may have overtreated events that they deemed to require therapy (traumatic events or joint/muscle pain), which might not have been true bleeds 
and might not have needed to be treated. With ongoing time on trial, patients likely had greater confidence in the level of prophylactic protection provided by N9-GP, which may have caused them to not treat events that initially they might have treated. Additional reasons for the decrease in bleeding rates include the gradually increasing FIX trough activity and possible improved joint health over time.

The median overall ABR reported in the 5-year analysis in our study (0.66) compares extremely favorably to ABRs reported with prophylaxis using standard half-life rFIX products in children (median overall ABR of 2.0 and estimated overall $A B R$ of 3.7, respectively). ${ }^{19,20}$ It also compares favorably with the median overall ABR reported for prophylaxis with rFIX Fc fusion protein in children over a median treatment duration of 1.1 to 3.4 years (ABR: 1.04-3.69) and recombinant fusion protein linking coagulation FIX with albumin (rIX-FP) administered for a mean duration of 62 weeks (ABR: 3.12). 21,22

During the first-year analysis of paradigm 5, 10 patients $(40.0 \%)$ had remained free from any bleeds. ${ }^{13}$ In other prophylaxis studies with EHL FIX products in patients with hemophilia B and FIX activity $\leq 2 \%$, outcomes were similar over an approximate 1-year treatment period. In a study of rFIX Fc fusion protein, 10 (33.0\%) patients remained free from bleeds over a median treatment period of 49.4 weeks, ${ }^{23}$ and $4(14.8 \%)$ patients receiving rIX-FP experienced no bleeds requiring treatment during a mean treatment period of 62 weeks. ${ }^{22}$ Over the entire 5 -year analysis period of paradigm 5, 5 (20\%) patients remained free from bleeds, while 16 patients (64\%) had no spontaneous bleeds. There are no similar long-term ABR data reported for other EHL FIX products.

The hemostatic success rate in the 5-year analysis of paradigm 5 was slightly lower than that observed in the 1year analysis ( $88.7 \%$ vs. $92.9 \%$ of bleeds $)^{13}$; to a large extent, this was due to a single patient who was noncompliant and who received up to 12 doses of N9-GP on several occasions to treat bleeds. Excluding this patient, $91.8 \%$ of all bleeds were successfully treated. In this trial, the majority (81.7\%) of bleeds were successfully treated with one N9-GP infusion, while $11.3 \%$ were treated with two infusions; only $7 \%$ of bleeds in four patients were managed with three or more N9-GP infusions.

These hemostatic success outcomes are similar to those reported in two shorter trials (paradigm 2 and paradigm 4 ) of weekly prophylactic N9-GP (40 IU/ $/ \mathrm{kg})$ in adolescents/adults with hemophilia B and FIX activity $\leq 2 \% .{ }^{10,11}$ In paradigm 2 , $95.7 \%$ of bleeds were successfully controlled, and $98.6 \%$ required only one N9-GP infusion to treat the bleeding episode. Corresponding values in paradigm 4 were 92.9 and $84.7 \%$, respectively. ${ }^{10,11}$

A limitation of this trial is its small sample size. Sample size is always a challenge when conducting clinical trials in rare diseases (such as hemophilia B) with low patient numbers. Furthermore, patients with mild (FIX activity: $5-40 \%)^{4}$ or moderate (FIX activity: $>2-5 \%$ ) hemophilia B were not eligible for inclusion in this trial, further reducing the sample size. Additionally, maintaining patients on long- term trials is challenging, particularly when the investigational product becomes available outside of a clinical trialas has happened with N9-GP, now available commercially in many of the participating centers/countries.

In conclusion, this trial provides the longest follow-up safety and efficacy data of any EHL FIX in children. Such longterm follow-up data are important, particularly in very young children who are growing and developing and who start off with good joint status. The data from this 5-year analysis of paradigm 5 substantiate the long-term safety and efficacy of N9-GP for the prevention and treatment of bleeds in previously treated children (aged $0-12$ years) with hemophilia B with FIX activity $\leq 2 \%$.

\section{What is known about this topic?}

- Long-term prophylaxis with replacement factor IX (FIX) concentrate is indicated for children with severe hemophilia B (HB). However, such prophylaxis is challenging due to the need for frequent intravenous FIX infusions.

- Nonacog beta pegol (N9-GP) is an extended half-life (EHL) glycoPEGylated FIX, which has demonstrated a fivefold prolongation of half-life versus standard halflife FIX. It has shown good safety and efficacy as prophylaxis in children with $\mathrm{HB}$.

- Few published data exist on the long-term safety and efficacy of prophylaxis with an EHL FIX such as N9-GP in children with $\mathrm{HB}$.

\section{What does this paper add?}

- This 5-year analysis of prophylactic N9-GP administered as $40 \mathrm{IU} / \mathrm{kg}$ once weekly provides the longest follow-up safety and efficacy data published of any EHL FIX.

- No neurological, renal, or hepatic safety concerns were identified, and no patients developed anti-FIX inhibitory antibodies; PEG plasma concentration reached steady state at 6 months, after which there was a slight increase proportional to the increased steady-state FIX trough activity (mean FIX trough activity in 7-12 year age group at 5 years: $19.2 \%$ ). Bleeding rates continued to decrease during the trial, such that over the entire 5 -year period, the median overall annualized bleeding rate was 0.66 .

- Data from this 5-year analysis of paradigm 5 substantiate the long-term safety and efficacy of N9-GP for the prevention and treatment of bleeds in previously treated children (aged 0-12) with HB with FIX activity $\leq 2 \%$.

Funding

This trial was sponsored by Novo Nordisk A/S (Bagsværd, Denmark). 


\section{Conflict of Interest}

M.C. has received grant/research support from: Baxalta (Shire), Bayer, Biogen, Novo Nordisk and Pfizer, has been a consultant for Baxalta/Shire/Takeda, Bayer, Biogen/Bioverativ/Sanofi, CSL Behring, Grifols, Novo Nordisk, Octapharma, Pfizer, and Roche, and is a member of speaker bureaus of Baxalta (Shire), Bayer, Biogen, CSL Behring, Grifols, Novo Nordisk, Octapharma, Pfizer, and Roche.

S.K. has received grant/research support from: local principal investigator (PI) research funding for Bayer, Bioverativ, Daiichi Sankyo, Grifols, and Novo Nordisk, and is a member of speaker bureaus/advisory boards for Bayer, Bioverativ, and Novo Nordisk.

M.Y.L. has no conflict of interest.

M.T. has served on advisory boards for Bayer, Bioverativ, Chugai, and Novo Nordisk; has received research support in clinical trials as a local PI for Bioverativ, Chugai, CSL Behring, and Novo Nordisk, and has received speaker's fees from Bayer, Bioverativ, Chugai, CSL Behring, Novo Nordisk, and Shire.

D.R. and C.S. are employees of Novo Nordisk A/S, Søborg, Denmark.

E.S. has served on advisory boards for Bayer, Bioverativ, CSL Behring, Grifols, Kedrion, Novo Nordisk, Octapharma, Pfizer, Roche, Shire/Takeda, SOBI, Spark, and Uniqure and has been a member of speaker bureaus for Bayer, CSL Behring, Grifols, Kedrion, Novo Nordisk, Pfizer, Roche, Shire/Takeda, and SOBI.

\section{Acknowledgments}

M. Watson of Parexel, a medical writer supported by funding by Novo Nordisk A/S, provided an initial draft and editorial assistance to the authors during preparation of this manuscript. Novo Nordisk's policy on data sharing may be found at https://www.novonordisk-trials.com/how-access-clinical-trial-datasets.

\section{References}

1 Castaman G. The benefits of prophylaxis in patients with hemophilia B. Expert Rev Hematol 2018;11(08):673-683

2 Witmer C, Pressley R, Kulkarni R, et al. Intracranial hemorrhage in patients with hemophilia in the prophylaxis era. Blood 2008;112 (11):3388-3388

3 Pencharz PB. Drug dosing in children-special considerations. Haemophilia 2006;12(04):30-32

4 World Federation of Hemophilia. 2012. Guidelines for the management of hemophilia. Available at: http://www1.wfh.org/publications/files/pdf-1472.pdf. Accessed July 22, 2019

5 National Hemophilia Foundation. 2016. MASAC Recommendation Concerning Prophylaxis. Available at: https://www.hemophilia.org/sites/default/files/document/files/241Prophylaxis.pdf. Accessed July 22, 2019

6 Ljung R. Aspects of prophylactic treatment of hemophilia. Thromb J 2016;14(Suppl 1):30
7 Hacker MR, Geraghty S, Manco-Johnson M. Barriers to compliance with prophylaxis therapy in haemophilia. Haemophilia 2001;7 (04):392-396

8 Santagostino E, Mancuso ME. Barriers to primary prophylaxis in haemophilic children: the issue of the venous access. Blood Transfus 2008;6(Suppl 2):s12-s16

9 Negrier C, Knobe K, Tiede A, Giangrande P, Møss J. Enhanced pharmacokinetic properties of a glycoPEGylated recombinant factor IX: a first human dose trial in patients with hemophilia B. Blood 2011;118(10):2695-2701

10 Collins PW, Young G, Knobe K, et al; paradigm 2 Investigators. Recombinant long-acting glycoPEGylated factor IX in hemophilia B: a multinational randomized phase 3 trial. Blood 2014;124(26): 3880-3886

11 Young G, Collins PW, Colberg T, et al. Nonacog beta pegol (N9-GP) in haemophilia B: a multinational phase III safety and efficacy extension trial (paradigm ${ }^{\mathrm{TM}} 4$ ). Thromb Res 2016;141:69-76

12 Escobar MA, Tehranchi R, Karim FA, et al. Low-factor consumption for major surgery in haemophilia B with long-acting recombinant glycoPEGylated factor IX. Haemophilia 2017;23(01):67-76

13 Carcao M, Zak M, Abdul Karim F, et al. Nonacog beta pegol in previously treated children with hemophilia $B$ : results from an international open-label phase 3 trial. J Thromb Haemost 2016;14 (08):1521-1529

14 Garcês S, Demengeot J, Canas-da-Silva J, et al. AB1297 bridging ELISA as a screening assay to monitor immunogenicity in routine clinical practice. Ann Rheum Dis 2013;71(Suppl 3):711

15 Lin AV. Direct ELISA. Methods Mol Biol 2015;1318:61-67

16 European Medicines Agency. Guideline on clinical investigation of recombinant and human plasma-derived factor IX products; Accessed July 22, 2019

17 Sternebring O, Gabel-Jensen C, Jacobsen H, Benie AJ, Bjørnsdottir I. Steady-state plasma concentrations of polyethylene glycol (PEG) are reached in children and adults during once-weekly prophylactic treatment with nonacog beta pegol (N9-GP). BioDrugs 2019;33(06):673-681

18 Stidl R, Denne M, Goldstine J, Kadish B, Korakas KI, Turecek PL. Polyethylene glycol exposure with antihemophilic factor (recombinant), PEGylated (rurioctocog alfa pegol) and other therapies indicated for the pediatric population: history and safety. Pharmaceuticals (Basel) 2018;11(03):e75

19 Monahan PE, Liesner R, Sullivan ST, Ramirez ME, Kelly P, Roth DA. Safety and efficacy of investigator-prescribed BeneFIX prophylaxis in children less than 6 years of age with severe haemophilia B. Haemophilia 2010;16(03):460-468

20 Urasinski T, Stasyshyn O, Andreeva T, et al. Recombinant factor IX (BAX326) in previously treated paediatric patients with haemophilia B: a prospective clinical trial. Haemophilia 2015;21(02): 196-203

21 Ragni M, Kulkarni R, Pasi J, et al. B-YOND final results confirm established safety, sustained efficacy, and extended dosing interval for up to 4 years of treatment with rFIXFc in previously treated subjects with severe hemophilia B. Paper presented at: ASH 2018

22 Kenet G, Chambost H, Male C, et al; PROLONG-9FP Investigator Study Group. Long-acting recombinant fusion protein linking coagulation factor IX with albumin (rIX-FP) in children. Results of a phase 3 trial. Thromb Haemost 2016;116(04):659-668

23 Fischer K, Kulkarni R, Nolan B, et al. Recombinant factor IX Fc fusion protein in children with haemophilia B (Kids B-LONG): results from a multicentre, non-randomised phase 3 study. Lancet Haematol 2017;4(02):e75-e82 